\title{
Hyperfine-induced valley mixing and the spin-valley blockade in carbon-based quantum dots
}

\author{
András Pályi and Guido Burkard \\ Department of Physics, University of Konstanz, D-78457 Konstanz, Germany
}

(Dated: June 15, 2018)

\begin{abstract}
Hyperfine interaction (HFI) in carbon nanotube and graphene quantum dots is due to the presence of ${ }^{13} \mathrm{C}$ atoms. We theoretically show that in these structures the short-range nature of the HFI gives rise to a coupling between the valley degree of freedom of the electron and the nuclear spin, in addition to the usual electron spin-nuclear spin coupling. We predict that this property of the HFI affects the Pauli blockade transport in carbon-based double quantum dots. In particular, we show that transport is blocked only if both the spin and the valley degeneracies of the quantum dot levels are lifted, e.g., by an appropriately oriented magnetic field. The blockade is caused by four "supertriplet" states in the $(1,1)$ charge configuration.

PACS numbers: 73.63.Kv, 73.63.Fg, 73.23.Hk, 31.30.Gs
\end{abstract}

In the past decade, fundamental steps have been made towards the realization of quantum information processing, including isolation, manipulation, and readout of single electron spins in the solid state $\stackrel{1}{1}$ However, the majority of the existing quantum dot (QD) spin qubits is fabricated in material systems, where hyperfine interaction (HFI) with nuclear spins limits the device performance via spin decoherence. Carbon structures, such as carbon nanotubes (CNTs) or graphene, are expected to have weak HFI, due to the small $1 \%$ natural abundance of spin-carrying ${ }^{13} \mathrm{C}$ nuclei. This expectation has motivated intensive theoretical investigation $2,3,4,5$ and the experimental realization of QDs in carbon nanostructures $, 6.7,8,9,10,11,12,13,14,15 \mathrm{~A}$ further perspective of carbon-based quantum information processing has been opened by proposals suggesting to utilize the valley degree of freedom of the delocalized electrons as a qubit $\frac{16,17}{17}$ Relaxation and decoherence mechanisms of these valley-qubits are yet to be explored. One possible source of those is short-range disorder, which is known to couple the two different valley states, $\underline{18}$

Double quantum dot (DQD) structures in the twoelectron regime are particularly well suited for studying the effects of HFI $19,20,21,22,23$ In the so-called Pauli blockade regime, the measurement of the direct current through a serially coupled GaAs DQD, as a function of the external magnetic field, has been used to infer the hyperfine energy scale ${ }^{20,21}$ Another experiment in GaAs DQDs showed that HFI can be utilized to perform coherent rotations between the states of a singlet-triplet qubit and, at the same time, acts as a source of decoherence ${ }^{22}$

The effect of the HFI in carbon is most pronounced in fully ${ }^{13} \mathrm{C}$-enriched samples $\frac{5,9,10,24,25,26}{2}$ Such nanotube DQD devices have been used recently to estimate the energy scale of the atomic HFI as $\sim 100 \mu \mathrm{eV}$, using transport $\frac{10}{10}$ and singlet-triplet dephasing time $\mathrm{e}^{9}$ measurements. In contrast, theory predicts an atomic hyperfine energy scale $\sim 1 \mu \mathrm{eV} \stackrel{5,24}{ }$ This discrepancy between theory and experiment, together with unexplained features of the dephasing time measurements of Ref. 9 , show that additional theoretical efforts have to be made to gain a complete understanding of the role of HFI in carbonbased QDs.

Here, we study the influence of the ${ }^{13} \mathrm{C}$ nuclear spins on the spin and valley degrees of freedom of the electrons in carbon-based QDs. In particular, we derive the $4 \times$ 4 Hamiltonian describing the effect of HFI on a single fourfold (spin and valley) degenerate QD energy level. We find that due to the short-range nature of the HFI, it couples the nuclear spins not only with the spin, but also with the valley degree of freedom of the electron. The effective hyperfine Hamiltonian can be expressed as

$$
H_{\mathrm{hf}}=\boldsymbol{S} \cdot\left(\boldsymbol{h}^{(0)} \tau_{0}+\sum_{i=x, y, z} \boldsymbol{h}^{(i)} \tau_{i}\right) .
$$

Here $\boldsymbol{S}=\left(s_{x}, s_{y}, s_{z}\right) / 2$ is the spin operator, $\tau_{0}$ is the unit operator in valley space, $s_{i}\left(\tau_{i}\right)$ denotes the Pauli matrices acting in spin (valley) space, and the quantities $\boldsymbol{h}^{(0, x, y, z)}$ are different linear combinations of the individual nuclear spin operators (see below). Equation (1) should be contrasted with the widely used $2 \times 2$ hyperfine Hamiltonian $H_{\mathrm{hf}, \mathrm{GaAs}}=\boldsymbol{S} \cdot \boldsymbol{h}$, which describes the effect of the nuclear spin on a twofold degenerate level in a GaAs QD, and incorporates only a single Overhauser field $\boldsymbol{h}$. We estimate that the order of magnitude of the valley-conserving $\left(\sim \tau_{0}\right)$ and valley-mixing parts of $H_{\mathrm{hf}}$ are the same.

As a physical consequence of the valley coupling due to the HFI, we predict that the response of the Pauli blockade leakage current through a carbon-based DQD to an applied external magnetic field is remarkably different from the case of GaAs DQDs. In the Pauli or spin blockade regime, $\frac{1}{1}$ transport from the source to the drain through a serially coupled DQD occurs via the $(0,1) \rightarrow(1,1) \rightarrow(0,2) \rightarrow(0,1)$ cycle, $\left(n_{L}, n_{R}\right)$ denoting the charge state with $n_{L}\left(n_{R}\right)$ electrons in the left (right) QD [see Fig. 1 (inset)]. In a GaAs DQD, blocking of the current occurs when there is at least one twoelectron energy eigenstate in the $(1,1)$ charge configuration having a spin wave function, which is symmetric 
under particle exchange (i.e., a triplet). Due to HFI, this condition is achieved only in the presence of an external magnetic field, which splits two triplet states apart from the singlet state and prevents hyperfine-induced mixing of those ${ }^{20,21}$ In carbon-based QDs with fourfold level degeneracy, the current is blocked only if there is at least one energy eigenstate in the $(1,1)$ charge configuration with a combined spin-valley wave function which is symmetric under particle exchange ('supertriplet'). In order to distinguish this effect from the spin blockade in conventional DQDs, we call it the spin-valley blockade. We show that in contrast to GaAs, in carbon DQDs a spin (Zeeman) splitting is insufficient to maintain the blockade in the presence of HFI, which, however, can be recovered by simultaneously introducing spin and valley splittings.

We consider the lowest-lying orbital level of an electrostatically defined QD in monolayer graphene with a finite gap ${ }^{3}$ (Nevertheless, the concepts and the formalism we use are readily generalizable to other types of graphene or CNT QDs.) In the absence of nuclear spins and external magnetic field, this level is fourfold (spin and valley) degenerate. In the presence of an external magnetic field, both the spin and valley degeneracy can be split: an in-plane magnetic field causes only a spin splitting $\Delta_{s}=g_{e} \mu_{B} B$ via the Zeeman effect, whereas an out-of-plane $(z)$ component introduces a valley splitting $\Delta_{v}\left(B_{z}\right)$ as well $\stackrel{\underline{3}}{\underline{b}}$ We consider the regime, where the energy difference between the lowest-lying and the second orbital levels is much larger than $\Delta_{s}$ and $\Delta_{v}$. We describe the system using the tight-binding (TB) model. The TB wave functions, corresponding to the four sublevels of the lowest-lying orbital level and characterized by the spin and valley quantum numbers $s \in$ $(\uparrow, \downarrow) \equiv(+,-)$ and $v \in\left(K, K^{\prime}\right) \equiv(+,-)$, are $\left(\psi_{s v}\right)_{l \sigma}=$ $\sqrt{\Omega_{\text {cell }}} e^{i v \boldsymbol{K} \cdot \boldsymbol{r}_{l \sigma}} \Psi_{\sigma}^{(v)}\left(\boldsymbol{r}_{l \sigma}\right) \chi_{s}$. Here $\sigma \in\{A, B\}$ is the sublattice index, $l$ is the unit cell index, $\Omega_{\text {cell }}$ is the unit cell area, $\boldsymbol{r}_{l \sigma}$ is the position of the carbon atom on sublattice $\sigma$ in the $l$ th unit cell, and $\chi_{+}=(1,0)$ and $\chi_{-}=(0,1)$ are the two possible spin states. The four smoothly varying functions $\Psi_{\sigma}^{(v)}$ can be obtained by solving the Dirac-like envelope function equation,$\frac{3,27}{2}$ The functions $\Psi_{\sigma}^{(v)}$ and $\psi_{s v}$ are normalized: $\int d^{2} \boldsymbol{r}\left(\left|\Psi_{A}^{(v)}(\boldsymbol{r})\right|^{2}+\left|\Psi_{B}^{(v)}(\boldsymbol{r})\right|^{2}\right)=1$ and $\sum_{l \sigma}\left|\left(\psi_{s v}\right)_{l \sigma}\right|^{2}=1$.

The nuclear spin of the carbon atom on site $l \sigma$ is denoted by $\boldsymbol{I}_{l \sigma}$, being zero if the atom is a ${ }^{12} \mathrm{C}$ and a spin- $1 / 2$ operator if the atom is ${ }^{13} \mathrm{C}$. In graphene and CNTs, the HFI is known to be short-range: its major contribution to the TB Hamiltonian is the on-site matrix element on the site of the nuclear spin $\frac{\underline{5}}{\underline{n}}$ Therefore the elements of the TB Hamiltonian matrix, describing the HFI, are $\left(H_{\mathrm{hf}, \mathrm{tb}}\right)_{l \sigma, l^{\prime} \sigma^{\prime}}=\delta_{l l^{\prime}} \delta_{\sigma \sigma^{\prime}} \boldsymbol{S} A \boldsymbol{I}_{l \sigma}$, where $A=\operatorname{diag}\left(A_{x}, A_{y}, A_{z}\right)$ is a diagonal matrix.

The effective Hamiltonian describing the influence of the HFI on the four sublevels is constructed by expressing $H_{\mathrm{hf}, \mathrm{tb}}$ in the subspace spanned by the four TB wave functions $\psi_{s v}$. The resulting matrix can be expressed in

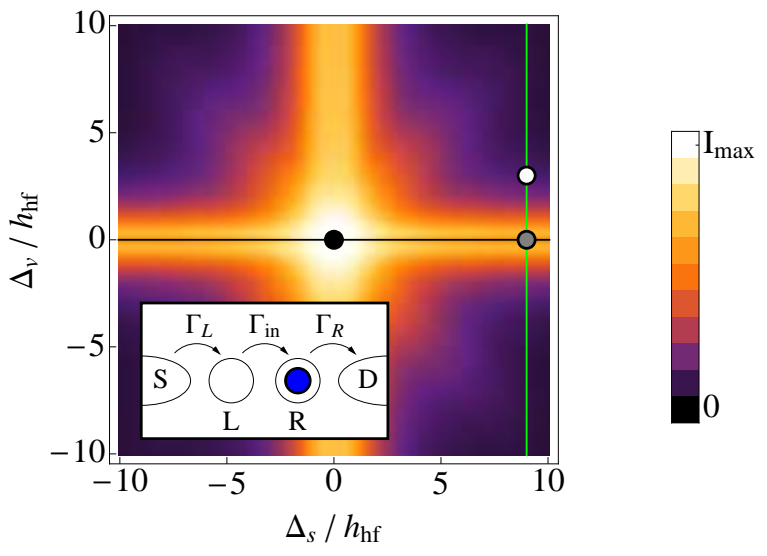

FIG. 1: (Color online) Averaged leakage current $\langle I\rangle$ due to hyperfine interaction in a graphene DQD, as a function of spin and valley splittings. $I_{\max } \approx 0.34 e \Gamma_{\text {in }}$. Inset: transport through a DQD. Dot $R$ is always occupied by at least one electron.

terms of the spin and valley operators as shown in Eq. (1) using

$$
\boldsymbol{h}^{(k)}=\Omega_{\mathrm{cell}} A \sum_{l \sigma} \boldsymbol{I}_{l \sigma} F_{l \sigma}^{(k)} \quad(k=0, x, y, z),
$$

where $F_{l \sigma}^{(0)}=\sum_{v} f_{l \sigma}^{(v)} / 2, F_{l \sigma}^{(z)}=\sum_{v} v f_{l \sigma}^{(v)} / 2, F_{l \sigma}^{(x / y)}=$ $\operatorname{Re} / \operatorname{Im}\left(e^{-2 i \boldsymbol{K} \cdot \boldsymbol{r}_{l \sigma}} g_{l \sigma}\right)$, with $f_{l \sigma}^{(v)}=\left|\Psi_{\sigma}^{(v)}\left(\boldsymbol{r}_{l \sigma}\right)\right|^{2}$ and $g_{l \sigma}=$ $\Psi_{\sigma}^{(+) *}\left(\boldsymbol{r}_{l \sigma}\right) \Psi_{\sigma}^{(-)}\left(\boldsymbol{r}_{l \sigma}\right)$. Note that in the presence of time reversal symmetry $\boldsymbol{h}^{(z)}=0$. Under normal conditions, the nuclear spins are completely randomized by thermal fluctuations, which implies that the components of the hyperfine fields have zero mean and their variances are

$$
\left\langle\left(h_{j}^{(k)}\right)^{2}\right\rangle=\Omega_{\text {cell }}^{2} A_{j}^{2} \frac{\nu}{4} \sum_{l \sigma}\left(F_{l \sigma}^{(k)}\right)^{2} \quad(k=0, x, y, z) .
$$

We have used $\left\langle\left(I_{l \sigma}\right)_{j}^{2}\right\rangle=\nu / 4$ and $\nu$ denotes the abundance of ${ }^{13} \mathrm{C}$ atoms in the QD. Using the slowly varying nature of the envelope functions, we find $\sum_{l \sigma}\left(F_{l \sigma}^{(x, y)}\right)^{2}=\frac{1}{2} \sum_{l \sigma}\left|g_{l \sigma}\right|^{2}$. In the $N \rightarrow \infty$ limit $(N$ is the number of atoms in the QD) the distributions of the hyperfine fields $h_{j}^{(k)}$ converge to Gaussians. Furthermore, $h_{j}^{(x)}$ and $h_{j}^{(y)}$ become independent from $h_{j}^{(0)}, h_{j}^{(z)}$ and each other.

To determine the variances of the hyperfine field precisely, one needs to know the envelope functions $\Psi_{\sigma}^{(v)}$. However, a simple estimation can be given using the assumption $\left|\Psi_{\sigma}^{(v)}\right|^{2} \approx 1 / \Omega_{\text {cell }} N$. This choice satisfies the normalization condition, and it results in $\left\langle\left(h_{j}^{(0)}\right)^{2}\right\rangle=$ $A_{j}^{2} \nu / 4 N,\left\langle\left(h_{j}^{(x / y)}\right)^{2}\right\rangle=A_{j}^{2} \nu / 8 N$ and $\left\langle\left(h_{j}^{(z)}\right)^{2}\right\rangle=0$. We will use these values in the following calculations.

The Hamiltonian $H_{\mathrm{hf}}$ in Eq. (1) shows that the HFI in carbon-based QDs results both in spin and valley mixing, and our estimates of the hyperfine field variances 
suggest that the valley-conserving and valley-mixing contributions in $H_{\mathrm{hf}}$ have the same order of magnitude. We emphasize that this result is due to the short-range nature of the HFI. We anticipate a similar result in silicon QDs, $\stackrel{28,29}{2}$ where electrons also possess a valley degree of freedom. We highlight three possible physical consequences of the valley-mixing nature of $H_{\mathrm{hf}}$. (i) HFI can be a source of valley relaxation and decoherence in 'valleytronics' devices, such as valley filters, valley valves 16 or QD valley qubits $\frac{17}{\underline{17}}$ (ii) Spin-orbit coupling in CNT QDs can split the fourfold degenerate ground state dot level into two doublets $\left(K \uparrow, K^{\prime} \downarrow\right.$ and $\left.K^{\prime} \uparrow, K \downarrow\right)$ at $B=0 . \underline{7}$ Spin-orbit coupling is stronger than the HFI; hence, a fully valley-conserving $H_{\mathrm{hf}}$ would not be able to cause mixing within or between the Kramers doublets. According to our result, $H_{\mathrm{hf}}$ is not valley-conserving and causes mixing within the Kramers doublet. This finding may facilitate the understanding of yet unexplained features of recent experiments on ${ }^{13} \mathrm{C}$ nanotubes $\frac{9,10}{10}$ (iii) As we show below, the valley-mixing character of the HFI introduces remarkable features in the behavior of the Pauli blockade leakage current through a carbon-based DQD.

Our goal is to calculate the average leakage current through a DQD in the Pauli blockade regime as a function of spin and valley splittings. To this end, we have generalized the master equation formalism used in Ref 21 . We focus on the parameter regime where the result Eq. (11) of Ref 21 is valid: (i) The energy detuning $\Delta \equiv$ $E(0,2)-E(1,1)$ between the $(0,2)$ and $(1,1)$ charge states is much larger than the coherent tunneling strength between the two dots $(t)$, the characteristic energy scale describing the HFI $\left[h_{\mathrm{hf}}=\sqrt{(\nu / 4 N) \sum_{j} A_{j}^{2}}\right]$, and the spin and valley splittings $\left(\Delta_{s}, \Delta_{v}\right)$. This condition suppresses the coherent tunneling between the dots; therefore, the hybridization between $(1,1)$ and $(0,2)$ charge states is negligible. (ii) The $(1,1) \rightarrow(0,2)$ transition is an energetically downhill inelastic tunneling process, characterized by the rate $\Gamma_{\text {in }}$. (iii) $t^{2} /|\Delta| \ll h_{\mathrm{hf}}$, which enables one to neglect the exchange splitting within $(1,1)$ charge states. (iv) $\Gamma_{L} \gg \Gamma_{R} \gg \Gamma_{\text {in }}$, where the rate $\Gamma_{L}\left[\Gamma_{R}\right]$ describes the $(0,1) \rightarrow(1,1)[(0,2) \rightarrow(0,1)]$ transition. (v) $\Gamma_{L}<h_{\mathrm{hf}}$, which enables one to use a classical master equation to describe the transport process. Studying the Pauli blockade problem under the above specified conditions is motivated by the fact that the theory for GaAs DQDs in this parameter regime has been successful in describing recent experimental results $\underline{\underline{20}}$ We also adopt the constant interaction feature of the model used in Ref 21 . Investigation of parameter regimes where the Coulomb interaction results in Wigner molecule formation ${ }^{30,31,32}$ is beyond the scope of this Rapid Communication.

Our approach deviates from the one used in Ref.21 in the form of the single-particle Hamiltonians of the two QDs, incorporating external magnetic field and HFI: $H_{D}=H_{D, \text { magn }}+H_{D, \text { hf }}$, where $D \in\{L, R\}$, $H_{D, \text { magn }}=g_{e} \mu_{B} \boldsymbol{S}_{D} \cdot \boldsymbol{B}+\Delta_{v}\left(B_{z}\right) \tau_{z, D} / 2$ and $H_{D, \mathrm{hf}}=$ $\boldsymbol{S}_{D} \cdot \sum_{i=0, x, y, z} \boldsymbol{h}_{D}^{(i)} \tau_{i, D}$. According to our above analy-

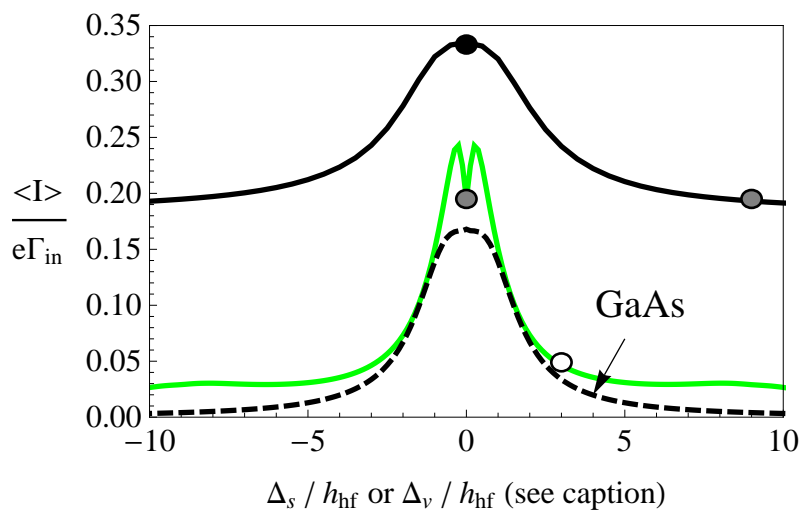

FIG. 2: (Color online) Averaged leakage current $\langle I\rangle$ as a function of (upper solid line, black) spin splitting $\Delta_{s}$ for zero valley splitting $\Delta_{v}=0$, (lower solid line, green) valley splitting $\Delta_{v}$ for $\Delta_{s}=9$, (dashed) $\Delta_{s}$ in a GaAs DQD [dashed line based on Eq. (11) of Ref 21].

sis, we treat $\boldsymbol{h}_{D}^{(0, x, y, z)}$ as uncorrelated stationary random fields ${ }^{21}$ and use our previous estimates for their variances. For our purposes, we can neglect the spin-anisotropy of the HFI,$\frac{5,24}{2}$ and use $A_{j}=A_{\text {iso }}$ and therefore $h_{\mathrm{hf}}=$ $A_{\text {iso }} \sqrt{3 \nu / 4 N}$. Evaluating $h_{\mathrm{hf}}$ with the measured ${ }^{9,10}$ value $A_{\text {iso }}=100 \mu \mathrm{eV}$ and assuming $N=7.5 \times 10^{4}$ atoms in a QD we find $h_{\mathrm{hf}} \approx 30 \mathrm{neV}(300 \mathrm{neV})$ for ${ }^{13} \mathrm{C}$ abundance $\nu=1 \%(100 \%)$; using the theoretical estimate ${ }^{5,24}$ $A_{\text {iso }}=1 \mu \mathrm{eV}$, we find $h_{\mathrm{hf}} \approx 0.3 \mathrm{neV}(3 \mathrm{neV})$. Under the above specified conditions, we set up a classical master equation for the occupation probabilities of the $(1,1)$ eigenstates of $H_{L}+H_{R}$. By solving the master equation numerically, we calculate the stationary current $I$ for many realizations of the nuclear fields and average those to obtain $\langle I\rangle$. In Fig. 1, we plot $\langle I\rangle$ as a function of spin and valley splittings obtained by averaging for 200 random realizations of the hyperfine fields. Two cuts along the horizontal (black) and vertical (green) lines of Fig. 1 are shown on Fig. 2, obtained by averaging over 3000 random realizations of the nuclear fields. For comparison, in Fig. 2 2 we also plotted the result corresponding to the case of GaAs DQDs (dashed).

One of the characteristic features of the spin-valley blockade is the cross-shaped pattern in the $\left\langle I\left(\Delta_{s}, \Delta_{v}\right)\right\rangle$ density plot in Fig. 1. This pattern indicates that the current is strongly suppressed only if both the spin and valley splittings $\left(\Delta_{s}\right.$ and $\left.\Delta_{v}\right)$ exceed the energy scale of the hyperfine coupling. As mentioned earlier, a physical situation where $\Delta_{s}$ is finite but $\Delta_{v}=0$, is when an in-plane magnetic field is applied to the system. This situation corresponds to the black line in Fig. 1 and the solid black curve $\left\langle I\left(\Delta_{s}\right)\right\rangle$ in Fig. 2. It is apparent from Fig. 2 that the current decreases with increasing $\Delta_{s}$; but instead of approaching zero, it saturates to a finite value around $0.2 e \Gamma_{\text {in }}$. This is in stark contrast to the case of GaAs DQDs, where $\Delta_{s}>h_{\mathrm{hf}}$ leads to a sharp decay of the current (dashed curve).

To present a qualitative interpretation of our results, 


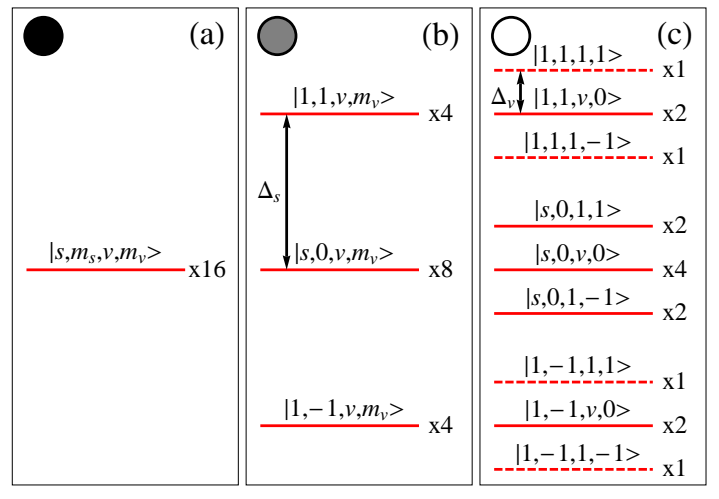

FIG. 3: (Color online) Schematic energy diagrams of the $(1,1)$ charge configuration corresponding to the three highlighted points of Fig. 11 The effect of hyperfine interaction is excluded. Dashed lines: transport-blocking supertriplet states.

we make use of the analogy between the spin and valley degrees of freedom. We describe the states of the $(1,1)$ charge configuration by using the simultaneous eigenbasis of the total spin operator $\left(\boldsymbol{S}_{L}+\boldsymbol{S}_{R}\right)^{2}$, the spin projection on the direction of the magnetic field $\left(\boldsymbol{S}_{L}+\boldsymbol{S}_{R}\right) \cdot \boldsymbol{B} / B$, the total valley operator $\left(\boldsymbol{\tau}_{L}+\boldsymbol{\tau}_{R}\right)^{2} / 4$, and the $z$-component of the valley operator $\left(\tau_{z, L}+\tau_{z, R}\right) / 2$. The corresponding quantum numbers are $s \in\{0,1\}, m_{s} \in\{-s, \ldots, s\}$, $v \in\{0,1\}$, and $m_{v} \in\{-v, \ldots, v\}$. We denote these basis states with $\left|s, m_{s}, v, m_{v}\right\rangle$. These are eigenstates of the system Hamiltonian in the absence of HFI. The combined spin-valley wave functions of the ten states fulfilling $s=v$ are supertriplets; therefore, these states cannot be squeezed into a $(0,2)$ charge configuration. In contrast, the spin-valley wave functions of the six states with $s \neq v$ are supersinglets; hence, their transition to $(0,2)$ is allowed.

The energy diagram of the 16 states of the $(1,1)$ charge configuration, corresponding to the three highlighted points of Fig. 1 are presented in Fig. 3. Figure 3a shows the situation, where $\Delta_{s}=\Delta_{v}=0$. In this case, there is a 16 -fold degenerate level, and the HFI mixes supersinglet and supertriplet states effectively. This results in a maximal current through the DQD. The Zeeman effect splits the states with different $m_{s}$ quantum numbers (Fig. 3 3 ) and suppresses hyperfine-induced hybridization between them if $\Delta_{s}>h_{\mathrm{hf}}$, which leads to a decrease in the leakage current. However, the valley mixing contribution of the HFI still induces strong mixing within the states with the same $m_{s}$. This mixing prevents the appearance of "pure" supertriplet energy eigenstates which would block the transport; therefore, the current does not drop to zero. As mentioned earlier, this behavior is in contrast to the case of GaAs DQDs. Figure 3r. shows the energy diagram when both $\Delta_{s}$ and $\Delta_{v}$ are finite. If those are larger than the HFI, then the four supertriplet states $|1, \pm 1,1, \pm 1\rangle$ become decoupled from supersinglets. Thus, the system gets trapped whenever any of these four states is occupied during the transport process, which results in a strong suppression of the current. Note that only two blocked states remain if $\Delta_{s} \approx \Delta_{v}$. In that case, $|1,1,1,-1\rangle$ and $|1,-1,1,1\rangle$ become degenerate with the fourfold degenerate $|s, 0, v, 0\rangle$ and mix with those due to HFI, slightly enhancing the current, visible along the diagonal $\left|\Delta_{s}\right|=\left|\Delta_{v}\right|$ lines in Fig. 1

Another characteristic of the spin-valley blockade is the appearance of a dip in the green $\left\langle I\left(\Delta_{v}\right)\right\rangle$ curve in Fig. 2 at $\Delta_{v}=0$. Similar dip structures have been predicted ${ }^{33,34}$ and measured $10,20,35$ in conventional semiconductors and they were attributed to various microscopic origins, including cotunneling, spin-orbit interaction, and exchange coupling. In our case, the dip has a different origin: it is due to the strong valley anisotropy of the HFI, i.e., that in our above estimations $h_{j}^{(z)}$ vanishes and therefore $H_{\mathrm{hf}}$ does not include the $\tau_{z}$ operator.

We have established the form of the Hamiltonian describing the effect of HFI on a fourfold degenerate energy level in a carbon-based QD. We have found that the short range nature of the HFI leads to a significant nuclear spin-electron valley coupling. We have calculated the effect of this interaction on the leakage current through a DQD in the Pauli blockade regime. Our findings may have profound consequences for both spin and valley manipulation in carbon-based QDs.

We thank K. Flensberg for a useful discussion, and DFG for financial support within SFB 767, SPP 1285, and FOR 912.
1 R. Hanson, L. P. Kouwenhoven, J. R. Petta, S. Tarucha, and L. M. K. Vandersypen, Rev. Mod. Phys. 79, 1217 (2007).

2 B. Trauzettel, D. V. Bulaev, D. Loss, and G. Burkard, Nat. Phys. 3, 192 (2007).

3 P. Recher, J. Nilsson, G. Burkard, and B. Trauzettel, Phys. Rev. B 79, 085407 (2009).

4 D. V. Bulaev, B. Trauzettel, and D. Loss, Phys. Rev. B 77, 235301 (2008).

5 J. Fischer, B. Trauzettel, and D. Loss, Phys. Rev. B 80, 155401 (2009).
6 M. R. Gräber, W. A. Coish, C. Hoffmann, M. Weiss, J. Furer, S. Oberholzer, D. Loss, and C. Schönenberger, Phys. Rev. B 74, 075427 (2006).

7 F. Kuemmeth, S. Ilani, D. C. Ralph, and P. L. McEuen, Nature 452, 448 (2008).

8 M. R. Buitelaar, J. Fransson, A. L. Cantone, C. G. Smith, D. Anderson, G. A. C. Jones, A. Ardavan, A. N. Khlobystov, A. A. R. Watt, K. Porfyrakis, and G. A. D. Briggs, Phys. Rev. B 77, 245439 (2008).

9 H. O. H. Churchill, F. Kuemmeth, J. W. Harlow, A. J. Bestwick, E. I. Rashba, K. Flensberg, C. H. Stwertka, 
T. Taychatanapat, S. K. Watson, and C. M. Marcus, Phys. Rev. Lett. 102, 166802 (2009).

${ }^{10}$ H. O. H. Churchill, A. J. Bestwick, J. W. Harlow, F. Kuemmeth, D. Marcos, C. H. Stwertka, S. K. Watson, and C. M. Marcus, Nat. Phys. 5, 321 (2009).

11 G. A. Steele, G. Gotz, and L. P. Kouwenhoven, Nature Nanotechnology 4, 363 (2009).

12 L. A. Ponomarenko, F. Schedin, M. I. Katsnelson, R. Yang, E. W. Hill, K. S. Novoselov, and A. K. Geim, Science 320, 356 (2008).

13 C. Stampfer, J. Güttinger, F. Molitor, D. Graf, T. Ihn, and K. Ensslin, Appl. Phys. Lett. 92, 012102 (2008).

14 F. Molitor, S. Dröscher, J. Güttinger, A. Jacobsen, C. Stampfer, T. Ihn, and K. Ensslin, Appl. Phys. Lett. 94, 222107 (2009).

15 X. Liu, J. B. Oostinga, A. F. Morpurgo, and L. M. Vandersypen, Phys. Rev. B 80, 121407(R) (2009).

16 A. Rycerz, J. Tworzydlo, and C. W. J. Beenakker, Nat. Phys. 3, 172 (2007).

17 P. Recher, B. Trauzettel, A. Rycerz, Y. M. Blanter, C. W. J. Beenakker, and A. F. Morpurgo, Phys. Rev. B 76, 235404 (2007).

18 T. Ando and T. Nakanishi, J. Phys. Soc. Jap. 67, 1704 (1998).

19 K. Ono, D. G. Austing, Y. Tokura, and S. Tarucha, Science 297, 1313 (2002).

${ }^{20}$ F. H. L. Koppens, J. A. Folk, J. M. Elzerman, R. Hanson, L. H. W. van Beveren, T. Vink, H. P. Tranitz, W. Wegscheider, L. P. Kouwenhoven, and L. M. K. Vandersypen, Science 309, 1346 (2005).
${ }^{21}$ O. N. Jouravlev and Y. V. Nazarov, Phys. Rev. Lett. 96, 176804 (2006).

22 J. R. Petta, A. C. Johnson, J. M. Taylor, E. A. Laird, A. Yacoby, M. D. Lukin, C. M. Marcus, M. P. Hanson, and A. C. Gossard, Science 309, 2180 (2005).

${ }^{23}$ W. A. Coish and D. Loss, Phys. Rev. B 72, 125337 (2005).

24 O. V. Yazyev, Nano Lett. 8, 1011 (2008).

25 B. Braunecker, P. Simon, and D. Loss, Phys. Rev. Lett. 102, 116403 (2009).

26 B. Dóra and F. Simon, Phys. Rev. Lett. 102, 197602 (2009).

27 C. W. J. Beenakker, Rev. Mod. Phys. 80, 1337 (2008).

28 N. Shaji, C. B. Simmons, M. Thalakulam, L. J. Klein, H. Qin, H. Luo, D. E. Savage, M. G. Lagally, A. J. Rimberg, R. Joynt, M. Friesen, R. H. Blick, S. N. Coppersmith, and M. A. Eriksson, Nat. Phys. 4, 540 (2008).

29 D. Culcer, L. Cywinski, Q. Li, X. Hu, and S. Das Sarma, Phys. Rev. B 80, 205302 (2009).

30 M. Roy and P. A. Maksym, Europhys. Lett. 86, 37001 (2009).

31 B. Wunsch, Phys. Rev. B 79, 235408 (2009).

32 A. Secchi and M. Rontani, Phys. Rev. B 80, 041404(R) (2009).

33 F. Qassemi, W. A. Coish, and F. K. Wilhelm, Phys. Rev. Lett. 102, 176806 (2009).

34 J. Danon and Y. V. Nazarov, Phys. Rev. B 80, 041301(R) (2009).

35 A. Pfund, I. Shorubalko, K. Ensslin, and R. Leturcq, Phys. Rev. Lett. 99, 036801 (2007). 